\title{
Relativistic effects in the scalar meson dynamics
}

\author{
R. Kamiński, L. Leśniak \\ Department of Theoretical Physics, \\ Institute of Nuclear Physics, PL 31-342 Kraków, Poland \\ and \\ J.-P. Maillet \\ Division de Physique Théorique, IPN, F-91406 Orsay, \\ France
}

August 20, 2018

\begin{abstract}
A separable potential formalism is used to describe the $\pi \pi$ and $K \bar{K}$ interactions in the $\mathrm{I}^{G}\left(\mathrm{~J}^{P C}\right)=0^{+}\left(0^{++}\right)$states in the energy range from the $\pi \pi$ threshold up to $1.4 \mathrm{GeV}$. Introduction of relativistic propagators into a system of Lippmann-Schwinger equations leads to a very good description of the data $\left(\chi^{2}=0.93\right.$ per one degree of freedom). Three poles are found in this energy region: $f_{0}(500)(M=506 \pm 10$ $\mathrm{MeV}, \Gamma=494 \pm 5 \mathrm{MeV}), f_{0}(975)(M=973 \pm 2 \mathrm{MeV}, \Gamma=29 \pm 2$ $\mathrm{MeV})$ and $f_{0}(1400)(M=1430 \pm 5 \mathrm{MeV}, \Gamma=145 \pm 25 \mathrm{MeV})$. The $f_{0}(975)$ state can be interpreted as a $K \bar{K}$ bound state. The $f_{0}(500)$ state may be associated with the often postulated very broad scalar resonance under the $K \bar{K}$ threshold (sometimes called $\sigma$ or $\epsilon$ meson). The scattering lengths in the $\pi \pi$ and $K \bar{K}$ channels have also been obtained. The relativistic approach provides qualitatively new results
\end{abstract}

*Unité de Recherche des Universités Paris 11 et Paris 6 associée au CNRS 
(for example the appearance of the $f_{0}(500)$ ) in comparison with previously used nonrelativistic approach. Interactions in both channels are attractive and have short range form factors.

PACS number(s): 14.40.Cs, 13.75.Lb, 12.40.Qq, 11.80.Gw

\section{Introduction}

Scalar meson spectroscopy is still far from being well understood [1-4]. Since it was difficult to explain known properties of the $\mathrm{I}^{G}\left(\mathrm{~J}^{P C}\right)=0^{+}\left(0^{++}\right)$ states using a standard $q \bar{q}$ picture, other models treating the four-quark states [5, 6] or meson-meson molecules [7-11] have been invented. There is also a continuous search [12, 13] for scalar gluonium states which can be mixed with the quark states of the same quantum numbers. The nature of the $f_{0}(975)$ and $\mathrm{a}_{0}(980)$ mesons is very controversial [14-17]. Closeness of their almost degenerated masses to the $K \bar{K}$ threshold energy constitutes an argument towards their interpretation as virtual bound $K \bar{K}$ states which are unstable due to the open $\pi \pi$ channel [10, 18-21].

In this article we extend the coupled channel formalism of Refs [18, 19] including some relativistic effects in both the $\pi \pi$ and $K \bar{K}$ decay channels of the scalar mesons. We use the relativistic propagators and the separable potentials in the Lippmann-Schwinger formalism. Such an approach has already been applied in the analysis of the pion-nucleon amplitudes [22] or in the studies of the $\Lambda(1405)$ resonance structure [23, 24]. Our aim is to describe quantitatively the isoscalar $s$-wave $\pi \pi$ and the $\bar{K} \bar{K}$ scattering data in a wide energy range starting from the $\pi \pi$ threshold up to $1.4 \mathrm{GeV}$. The parameters of the meson-meson interactions are fitted to the data and the $S$-matrix structure is analysed in order to extract information about the scalar resonances in that energy region. This is a new step in comparison with [18] where some pole structure of the $S$-matrix has been postulated and the $\chi^{2}$ tests have not been done. We observe that the relativistic effects are important not only in the $\pi \pi$ channel but also in the $K \bar{K}$ channel even near the threshold. This fact may have important implications, for example the $f_{0}(975)$ meson, interpreted as a $K \bar{K}$ molecule, can still have a relatively small radius (less than $1 \mathrm{fm}$ ). Consequently, the predictions of the radiative $\phi$ decay into the $K \bar{K} \gamma$ system via $\phi \rightarrow f_{0}(975) \gamma$ have to be substantially 
influenced [25]. The importance of the relativistic effects on the two photon decays of the $\mathrm{J}^{P C}=0^{++}$and $2^{++}$states has also been recently stressed [26].

This paper is organized as follows. In Sect. II we define our formalism. Sect. III is devoted to an analysis of the single $\pi \pi$ channel essentially up to the energy of about $700 \mathrm{MeV}$. The properties of the $K \bar{K}$ channel interaction in the decoupled case are discussed in Sect. IV. Both the nonrelativistic and relativistic cases are treated. In Sect. $\mathrm{V}$ we analyse the interactions in the coupled $\pi \pi$ and $K \bar{K}$ channels. A comparison with the data is performed and the $S$-matrix structure is studied. In Sect. VI we summarize the main results and outline some perspectives.

\section{The Formalism}

We describe the $\pi \pi$ and $K \bar{K}$ isoscalar $s$-wave interactions in the framework of the coupled channel Lippmann-Schwinger equations [22]. The scattering amplitude $T$ satisfies the following equation in the momentum space:

$$
\begin{aligned}
<\mathbf{p}|T| \mathbf{q}>= & <\mathbf{p}|V| \mathbf{q}>+ \\
& \int \frac{d^{3} s}{(2 \pi)^{3}}<\mathbf{p}|V| \mathbf{s}><\mathbf{s}|G| \mathbf{s}><\mathbf{s}|T| \mathbf{q}>,
\end{aligned}
$$

where $V, G, T$ are $2 \times 2$ matrices (label 1 denotes the $K \bar{K}$ channel and label 2 - the $\pi \pi$ channel), $V$ is the interaction matrix and $G$ is the diagonal matrix of propagators written in the center of mass system:

$$
<\mathbf{s}\left|G_{i j}\right| \mathbf{s}>=G_{i}(s) \delta_{i j} \quad(i, j=1,2),
$$

and $G_{i}^{-1}(s)=E-2 E_{i}(s)+i \epsilon, \epsilon \longrightarrow 0(+)$. In Eq. (2) $\mathbf{s}$ is the relative

momentum, $E$ is the total energy and $E_{i}(s)=\sqrt{s^{2}+m_{i}^{2}}$ are the relativistic energies; $m_{1}=495.69 \mathrm{MeV}, m_{2}=137.27 \mathrm{MeV}$ are the average kaon and pion masses.

A very convenient parameterization of the interaction matrix is a separable potential form. In the $K \bar{K}$ channel we use the simplest rank-one potential in the momentum space

$$
<\mathbf{p}\left|V_{11}\right| \mathbf{q}>=\lambda_{11} g_{1}(p) g_{1}(q)
$$


and in the $\pi \pi$ channel a rank-two potential

$$
<\mathbf{p}\left|V_{22}\right| \mathbf{q}>=\lambda_{22} g_{2}(p) g_{2}(q)+\lambda_{33} g_{3}(p) g_{3}(q)
$$

The transition potential matrix elements read

$$
<\mathbf{p}\left|V_{12}\right| \mathbf{q}>=<\mathbf{q}\left|V_{21}\right| \mathbf{p}>=\lambda_{12} g_{1}(p) g_{2}(q)+\lambda_{13} g_{1}(p) g_{3}(q) \text {. }
$$

In Eqs. (3)-(5) $\lambda_{i k}(i, k=1,2,3)$ are the coupling constants and $g_{i}$ are the form factors which we have chosen in the Yamaguchi form [27]:

$$
g_{i}(p)=\sqrt{\frac{4 \pi}{m_{i}}} \frac{1}{p^{2}+\beta_{i}^{2}},
$$

where

$$
m_{i}=\left\{\begin{array}{lll}
m_{K} & \text { if } & \mathrm{i}=1 \\
m_{\pi} & \text { if } & \mathrm{i}=2,3
\end{array}\right.
$$

and $\beta_{i}$ are the form factor range parameters. The potentials (3 -5) are nonlocal. After this choice the potential matrix has eight parameters (five coupling constants and three range parameters) which should be fixed by a comparison of the theoretical amplitudes with experimental data.

The separable form of the interactions enables us to write the following scattering matrix elements:

$$
\begin{aligned}
<\mathbf{p}\left|T_{11}\right| \mathbf{q}> & =g_{1}(p) t_{11} g_{1}(q) \\
<\mathbf{p}\left|T_{22}\right| \mathbf{q}> & =g_{2}(p) t_{22} g_{2}(q)+g_{2}(p) t_{23} g_{3}(q) \\
& +g_{3}(p) t_{32} g_{2}(q)+g_{3}(p) t_{33} g_{3}(q) \\
<\mathbf{p}\left|T_{12}\right| \mathbf{q}> & =<\mathbf{q}\left|T_{21}\right| \mathbf{p}>=g_{1}(p) t_{12} g_{2}(q)+g_{1}(p) t_{13} g_{3}(q),(10)
\end{aligned}
$$

where $t_{k l}$ are energy dependent reduced amplitudes $(k, l=1,2,3)$. In Eq. (8) $T_{11}$ denotes the $K \bar{K}$ elastic scattering amplitude, $T_{22}$ in Eq. (9) is the $\pi \pi$ elastic scattering amplitude while $T_{12}$ and $T_{21}$ are the transition $K \bar{K} \rightarrow \pi \pi$ and $\pi \pi \rightarrow K \bar{K}$ amplitudes. The system (11) of the coupled integral equations satisfied by the $T_{k l}$ elements leads to a set of algebraic equations for the reduced amplitudes $t_{k l}$ written in the $3 \times 3$ matrix form:

$$
t=\lambda+\lambda I t
$$


In Eq. (11) $\lambda$ is the symmetric $3 \times 3$ matrix of the coupling constants

$$
\lambda=\left(\begin{array}{ccc}
\lambda_{11} & \lambda_{12} & \lambda_{13} \\
\lambda_{12} & \lambda_{22} & 0 \\
\lambda_{13} & 0 & \lambda_{33}
\end{array}\right)
$$

and $I$ is the following symmetric matrix

$$
I=\left(\begin{array}{ccc}
I_{11} & 0 & 0 \\
0 & I_{22} & I_{23} \\
0 & I_{23} & I_{33}
\end{array}\right)
$$

consisting of the integrals

$$
I_{11}=\int \frac{d^{3} s}{(2 \pi)^{3}} g_{1}(s) G_{1}(s) g_{1}(s)
$$

and

$$
I_{k l}=\int \frac{d^{3} s}{(2 \pi)^{3}} g_{k}(s) G_{2}(s) g_{l}(s), \quad(k, l=2,3) .
$$

In Eqs. (66), (12) and (13) label 1 refers to the $K \bar{K}$ channel and labels 2 and 3 to the $\pi \pi$ channel in which the interaction potential contains two terms. The matrices $t$ and $I$ are functions of the energy $E$ (see Eq. (2)).

A solution of Eq. (11) is straightforward:

$$
t=(1-\lambda I)^{-1} \lambda
$$

and the resulting $t$-matrix is symmetric $\left(t_{k l}=t_{l k}\right)$ since the matrices $\lambda$ and $I$ are symmetric. Explicit expressions for the matrix elements $t_{k l}$ are given in the Appendix. All the functions $t_{k l}$ are inversely proportional to the Jost function

$$
D(E)=\operatorname{det}(1-\lambda I) .
$$

Let us notice that the coupling constants $\lambda_{i j}$ defined by (3)-(5) have the dimension $(\mathrm{MeV} / \mathrm{c})^{3}$ and the integrals (14 15) - the dimension $(\mathrm{MeV})^{-3}$. For simplicity in further calculations we will use the dimensionless coupling constants defined as

$$
\Lambda_{i j}=\frac{\lambda_{i j}}{2\left(\beta_{i} \beta_{j}\right)^{3 / 2}}
$$


and redefined integrals

$$
J_{i j}=2\left(\beta_{i} \beta_{j}\right)^{3 / 2} I_{i j} .
$$

If the energy $E$ is higher than the $K \bar{K}$ threshold mass we can relate (compare [22]) the on-shell scattering matrix elements $T_{k l}\left(k_{1}, k_{2}\right)$ to the $S$-matrix elements

$$
\begin{aligned}
& S_{11}=1-\frac{i k_{1} E_{1}\left(k_{1}\right)}{2 \pi} T_{11}\left(k_{1}, k_{2}\right), \\
& S_{22}=1-\frac{i k_{2} E_{2}\left(k_{2}\right)}{2 \pi} T_{22}\left(k_{1}, k_{2}\right), \\
& S_{12}=S_{21}=-\frac{i}{2 \pi} \sqrt{k_{1} E_{1}\left(k_{1}\right) k_{2} E_{2}\left(k_{2}\right)} T_{12}\left(k_{1}, k_{2}\right),
\end{aligned}
$$

where the $K \bar{K}$ channel and $\pi \pi$ channel momenta $k_{1}$ and $k_{2}$ are defined by the energy conservation condition:

$$
E=2 \sqrt{k_{1}^{2}+m_{K}^{2}}=2 \sqrt{k_{2}^{2}+m_{\pi}^{2}} .
$$

This relation allows one to write the functions $I_{11}(E)$ and $I_{k l}(E)$ appearing in Eqs. (14) and (15) as functions $I_{11}\left(k_{1}\right)$ and $I_{k l}\left(k_{2}\right)$. Similarly the Jost function $D(E)$ given by (17) can be expressed as a function of two related variables

$$
D\left(k_{1}, k_{2}\right)=D_{K}\left(k_{1}\right) D_{\pi}\left(k_{2}\right)-C\left(k_{1}, k_{2}\right),
$$

where

$$
\begin{aligned}
D_{K}\left(k_{1}\right) & =1-\Lambda_{11} J_{11}\left(k_{1}\right) \\
D_{\pi}\left(k_{2}\right) & =1-\Lambda_{22} J_{22}\left(k_{2}\right)-\Lambda_{33} J_{33}\left(k_{2}\right)+\Lambda_{22} \Lambda_{33} d\left(k_{2}\right), \\
C\left(k_{1}, k_{2}\right) & =\Lambda_{12} J_{11}\left(k_{1}\right)\left[\Lambda_{12} J_{22}\left(k_{2}\right)+\Lambda_{13} J_{23}\left(k_{2}\right)-\Lambda_{12} \Lambda_{33} d\left(k_{2}\right)\right] \\
& +\Lambda_{13} J_{11}\left(k_{1}\right)\left[\Lambda_{12} J_{23}\left(k_{2}\right)+\Lambda_{13} J_{33}\left(k_{2}\right)-\Lambda_{13} \Lambda_{22} d\left(k_{2}\right)\right]
\end{aligned}
$$

and

$$
d\left(k_{2}\right)=J_{22}\left(k_{2}\right) J_{33}\left(k_{2}\right)-J_{23}^{2}\left(k_{2}\right) .
$$

In Eq. (25) $D_{K}\left(k_{1}\right)$ is a part of the Jost function corresponding to the interaction in the single $K \bar{K}$ channel, $D_{\pi}\left(k_{2}\right)$ is a similar part in the $\pi \pi$ channel and $C\left(k_{1}, k_{2}\right)$ comes from the interchannel coupling. 
Using the Jost function $D$ we can also express the diagonal $S$-matrix elements as the ratios:

$$
\begin{aligned}
& S_{11}=\frac{D\left(-k_{1}, k_{2}\right)}{D\left(k_{1}, k_{2}\right)}, \\
& S_{22}=\frac{D\left(k_{1},-k_{2}\right)}{D\left(k_{1}, k_{2}\right)},
\end{aligned}
$$

and the nondiagonal matrix element satisfies the equation

$$
S_{11} S_{22}-S_{12}^{2}=\frac{D\left(-k_{1},-k_{2}\right)}{D\left(k_{1}, k_{2}\right)}
$$

Above the $K \bar{K}$ threshold the $S$-matrix can be parameterized in terms of the inelasticity parameter $\eta$ and the phase shifts $\delta_{\pi \pi}$ and $\delta_{K \bar{K}}$ :

$$
S=\left(\begin{array}{cc}
\eta e^{2 i \delta_{K} \bar{K}} & i \sqrt{1-\eta^{2}} e^{i\left(\delta_{\pi \pi}+\delta_{K \bar{K}}\right)} \\
i \sqrt{1-\eta^{2}} e^{i\left(\delta_{\pi \pi}+\delta_{K \bar{K}}\right)} & \eta e^{2 i \delta_{\pi \pi}}
\end{array}\right) .
$$

If the energy $E$ is lower than $2 m_{K}$ then we can still use Eqs. (21) and (30),

provided $k_{1}$ is purely imaginary $k_{1}=i \sqrt{m_{K}^{2}-E^{2} / 4}$. In this case the inelasticity parameter $\eta \equiv 1$ since the $K \bar{K}$ channel is closed.

Energy dependence of the experimentally measured quantities $\delta_{\pi \pi}, \delta_{K \bar{K}}$ and $\eta$ is closely related to the analytical structure of the Jost function in the complex planes of the $k_{1}$ and $k_{2}$ momenta.

\section{III $\pi \pi$ channel interactions}

In this section we consider interactions in the single $\pi \pi$ channel i.e. without couplings to the $K \bar{K}$ channel $\left(\lambda_{12}=\lambda_{13}=0\right)$. We study the energy range from the $\pi \pi$ threshold up to about $700 \mathrm{MeV}$, where in the first step the influence of the coupling to the $K \bar{K}$ channel can be neglected. The aim of such an analysis is an estimation of the $\pi \pi$ potential parameters and investigation of the pole structure of the $\pi \pi$ scattering function $S_{22}$. Two experimental facts can be quantitatively described in such an approach: the first is a positive value of the $\pi \pi s$-wave scattering length in the $I=0$ $s$-state, and the second is a systematic increase of the $\pi \pi$ phase shifts from 0 
degrees at the $\pi \pi$ threshold up to about 70 degrees at the energy of $700 \mathrm{MeV}$. Experimental data indicate the existence of the $f_{0}(1400)$ meson. Although the $f_{0}(1400)$ mass is higher than the energy limit under consideration, this resonance should be included in our analysis because of its large coupling to the $\pi \pi$ channel and a large width.

At the beginning let us discuss a case when the propagator $G_{2}$ (see Eq. (21)) has the nonrelativistic form $G_{2}^{-1}(s)=\left(k_{2}^{2}-s^{2}\right) / m_{2}+i \epsilon$. We start by assuming that in the potential $V_{22}$ in Eq. (四) only the first term, i.e. the coupling constant $\lambda_{33}=0$, is present. Then the Jost function $D_{\pi}\left(k_{2}\right)$ can be expressed as (see Eqs. (26) and (A13) in Appendix)

$$
D_{\pi}\left(k_{2}\right)=1+\frac{\Lambda_{22}}{\left(1-i a_{2}\right)^{2}}
$$

where $a_{2}=k_{2} / \beta_{2}$. Its phase is directly related to the phase shift $\delta_{\pi \pi}$ by

$$
D_{\pi}\left(k_{2}\right)=\left|D_{\pi}\left(k_{2}\right)\right| e^{-i \delta_{\pi \pi}} .
$$

Knowing that the scattering length

$$
a_{\pi \pi}=\lim _{k_{2} \longrightarrow 0} \delta_{\pi \pi} / k_{2}
$$

we can evaluate a low energy limit of the Jost function (33). As a result we obtain

$$
a_{\pi \pi}=-\frac{2 \Lambda_{22}}{\beta_{2}\left(1+\Lambda_{22}\right)} .
$$

The positivity of the experimental $a_{\pi \pi}$ value imposes the following conditions on the coupling constant $\Lambda_{22}$ :

$$
-1<\Lambda_{22}<0
$$

The analytical structure of the Jost function in Eq. (33) is very simple: it has a double pole at $k_{2}=-i \beta_{2}$ and two single roots at $k_{2}=i \beta_{2}\left( \pm \sqrt{-\Lambda_{2}}-1\right)$. For the $\Lambda_{22}$ satisfying the inequalities (37) both zeros lie on the negative part of the imaginary $k_{2}$-axis. Since the matrix element $S_{22}$ is given by a ratio $D_{\pi}\left(-k_{2}\right) / D_{\pi}\left(k_{2}\right)$, its poles coincide with the Jost function zeros of the denominator except of the numerator double pole at $k_{2}=i \beta_{2}$. The existence of the $f_{0}(1400)$ meson must manifest itself by the presence of two complex 
conjugated $S$-matrix poles lying off the imaginary $k_{2}$-axis. It has been shown in [18] that the introduction of the second term in the potential $V_{\pi \pi}$ permits one to obtain such a pair of poles and, in addition, a positive value of the scattering length. The coupling constants $\Lambda_{22}$ and $\Lambda_{33}$ in [18] had opposite signs. Although it was not shown there, we can prove that in the case when the $\pi \pi$ potential contains in Eq. (4) two terms, when there is no coupling to the $K \bar{K}$ channel, and the scattering length is positive, the two other poles of the $S_{22}$ can lie only on the imaginary axis.

Next we discuss the relativistic form of the propagator $G_{2}$ and investigate the analytical structure of the matrix element $S_{\pi \pi}$ calculated for a single term in $V_{\pi \pi}\left(\lambda_{33}=0\right)$. The situation now is quite different: the $S_{22}$ function has a pair of poles off the imaginary axis and simultaneously the condition $a_{\pi \pi}>0$ can be fulfilled. The expression for the scattering length in the relativistic case is

$$
a_{\pi \pi}=-\frac{2 \Lambda_{22}}{\beta_{2}\left\{1+\Lambda_{22}\left[\frac{1}{2}+\frac{1}{\pi}\left(\frac{\beta_{2}}{m_{2}}+\frac{m_{2}}{\beta_{2}} d_{2}\right)\right]\right\}},
$$

where $d_{2}$ is a constant defined in Appendix by (A12) and (A8). In order to get $a_{\pi \pi}>0$ the coupling constant $\Lambda_{22}$ must satisfy the following conditions:

$$
-\left[\frac{1}{2}+\frac{1}{\pi}\left(\frac{\beta_{2}}{m_{2}}+\frac{m_{2}}{\beta_{2}} d_{2}\right)\right]^{-1}<\Lambda_{22}<0 .
$$

Negative sign of the coupling constant $\Lambda_{22}$ means that the interaction is attractive.

Using (34) we have compared our theoretical predictions for the $\pi \pi$ phase shifts with the experimental data in the energy range from the $\pi \pi$ threshold up to $700 \mathrm{MeV}$. We have used the data from the $K_{e 4}$ decay 28] (5 points) and from the reaction $\pi^{-} p \rightarrow \pi^{-} \pi^{+} n$ [29-31] (16 points up to $700 \mathrm{MeV}$ ). We have performed the $\chi^{2}$ test using the CERN program MINUIT and as the output parameters we obtained $\Lambda_{22}=-0.213$ and $\beta_{2}=1239 \mathrm{MeV}$. For these parameters $\chi^{2}=1.13$ per one degree of freedom. The calculated scattering length $a_{\pi \pi}=0.18 \mathrm{~m}_{\pi}^{-1}$ can be compared with experimental values $(0.28 \pm 0.05) m_{\pi}^{-1}$ [28], $(0.24 \pm 0.09) m_{\pi}^{-1}$ [29] and $(0.207 \pm 0.028) m_{\pi}^{-1}$ [32]. In a combined analysis [33] of the $\pi N \rightarrow \pi \pi N$ data the value $a_{\pi \pi}=(0.197 \pm$ $0.01) m_{\pi}^{-1}$ is quoted. The scattering $S$-matrix function $S_{22}$ has only one pair of the complex conjugated poles at the energy $E=491 \pm i 246 \mathrm{MeV}$. It is 
clear that it cannot be related to the $f_{0}(1400)$ meson which according to the Particle Data Group [34] has the energy $1400-i(75 \div 200) \mathrm{MeV}$. In order to include the $f_{0}(1400)$ state in a further analysis we have added a second term to the $\pi \pi$ potential and the Jost function $D_{\pi}\left(k_{2}\right)$ obtained the form (26). Then in the complex momentum plane we have found a second pair of the complex conjugated poles of the $S_{22}$ function which can be related to the $f_{0}(1400)$ meson. In the numerical calculations of the pole positions we must take into account the energy range above $1 \mathrm{GeV}$ and include the coupling to the $K \bar{K}$ channel. Therefore a full discussion concerning the $f_{0}(1400)$ meson is presented in Section V. It is worth-while to note, however, that contrary to the nonrelativistic case, both coupling constants $\Lambda_{22}$ and $\Lambda_{33}$ have received negative signs.

The appearance of a new $S_{22}$ pole, in comparison with the nonrelativistic case, at an energy about $500 \mathrm{MeV}$ can be related to the existence of the socalled $\sigma$ or $\epsilon$ meson which has been often postulated both experimentally and theoretically (see references in [35]). The mass of this meson varies between about $500 \mathrm{MeV}$ and $1000 \mathrm{MeV}$, while the width even more - between 300 $\mathrm{MeV}$ and $1000 \mathrm{MeV}$. Various models of the $\sigma$ meson structure have been formulated describing it as normal $q \bar{q}, q q \overline{q q}$ or gluon-gluon states. Such a low mass scalar meson also appeared in the so-called $\sigma$-model and in other field theoretical models (see [36] and references cited therein) in which its mass had a value of about $500 \mathrm{MeV}$ and its large width varied from $300 \mathrm{MeV}$ to more than $500 \mathrm{MeV}$. The exchange of the $\sigma$ meson has been used in the Bonn model of the nucleon-nucleon interactions [37. It has been found that the $\sigma$ meson exchange can effectively describe the contribution of two correlated $s$-wave pions in the isospin 0 state. The $\sigma$ mass and width have been also recently discussed in the Nambu-Jona-Lasinio model [38]. An experimental measurement of the $\sigma$ parameters is very difficult because of its large width [35-39]. This meson does not appear as a typical Breit-Wigner peak and in the partial wave analyses it can easily be interpreted as a background. The last time when the $\sigma$ meson was included in the Particle Data Group Tables was 1974 with $M_{\sigma} \leq 700 \mathrm{MeV}$ and $\Gamma_{\sigma} \geq 600 \mathrm{MeV}$ (and it was called $\epsilon$ ) [39].

In the isospin 2 , spin $0 \pi \pi$ channel one does not expect a resonant behaviour of the scattering amplitude. Using a simple rank-one potential of the Yamaguchi form we have obtained a very good description of the corresponding $\pi \pi$ phase shifts up to about $1 \mathrm{GeV}$. No poles of the $\mathrm{I}=2$ scattering amplitude have been found in the momentum range of $R e k_{2}$ between 0 and 
$4 \mathrm{GeV}$ and $I m k_{2}$ between 0 and $-4 \mathrm{GeV}$. This fact, which confirms the above expectation, is in contrast to the situation in the isospin 0 channel in which we do find resonances.

\section{IV $K \bar{K}$ interactions}

Let us now discuss the $K \bar{K}$ interactions without coupling to the $\pi \pi$ channel. We shall be especially interested in the energy region close to the $K \bar{K}$ threshold near $1 \mathrm{GeV}$. In this paper we do not distinguish the $K^{0} \overline{K^{0}}$ and $K^{+} K^{-}$thresholds so we use their average energy value $E_{t h}=2 m_{1}=991.38$ $\mathrm{MeV}$. The $f_{0}(975)$ meson energy is very close to this value, so it is quite natural to interpret this state as a bound $K \bar{K}$ state with a binding energy of about $16 \mathrm{MeV}$. We do not, however, postulate the existence of the bound state from the beginning. As we shall see in the next chapter, the parameters of the $K \bar{K}$ interaction will be fixed by a global fit to the $\pi \pi$ and $K \bar{K}$ data. Here we discuss different conditions for the existence of the particular $K \bar{K}$ structures: bound states, antibound states (sometimes called virtual states [40]), or resonances. For each case we shall examine effects of the relativistic propagation of kaons and their influence on some observables, like scattering length or phase shifts. A very good method to distinguish the three types of the $K \bar{K}$ states is to look at the positions of the $S$-matrix poles corresponding to the Jost function zeros in the complex $k_{1}$-plane (Eq. 29). If the zero lies on the positive imaginary axis $\left(k_{1}=i \alpha_{1}, \alpha_{1}>0\right)$, then a bound state exists. The antibound state corresponds to a zero lying on the negative part of imaginary axis $\left(\alpha_{1}<0\right)$. If there are two complex conjugated zeros in the lower half plane then there is a resonance in the $K \bar{K}$ channel.

At first we analyse the most important features of the bound state. Its binding energy can be defined as

$$
E_{B}=2 m_{K}-2 \sqrt{m_{K}^{2}-\alpha_{1}^{2}} \quad \text { for } \quad \alpha_{1}<m_{K} .
$$

The Jost function $D_{K}\left(k_{1}\right)$ in Eq. (25) vanishes if

$$
\Lambda_{11}=1 / \operatorname{Re}\left[J_{11}\left(i \alpha_{1}\right)\right]
$$

since $\operatorname{Im}\left[J_{11}\left(i \alpha_{1}\right)\right] \equiv 0$. The coupling constant depends on the range pa-

rameter $\beta_{1}$ which can be chosen regardless of the binding energy $E_{B}$. In the 
nonrelativistic case we approximate the propagator $G_{1}$ in Eq. (2) by

$$
G_{1}(s)=\frac{m_{K}}{k_{1}^{2}-s^{2}} .
$$

Then according to Eq. (A13) the dimensionless $K \bar{K}$ coupling constant is particularly simple

$$
\Lambda_{11}=-\left(1+\frac{\alpha_{1}}{\beta_{1}}\right)^{2} .
$$

The $K \bar{K}$ force is attractive and the coupling constant is negative and smaller than -1 . For the relativistic propagator we have numerically verified (see also (Eq. A9)) that the $\beta_{1}$-dependence of $\Lambda_{11}$ is very similar to the one in the nonrelativistic case for the small values of $\beta_{1}$ up to about $200 \mathrm{MeV}$. For larger $\beta_{1}$ the absolute value of the "relativistic" coupling constant is smaller than the coupling constant in the nonrelativistic limit. If $\beta_{1} \longrightarrow \infty$, then nonrelativistically $\Lambda_{11} \longrightarrow-1$, while in the relativistic case $\Lambda_{11} \longrightarrow 0$ as $-\pi m_{K} / \beta_{1}$ (see Eqs. (A9) and (41)). This is the first indication that in the limit of large $\beta_{1}$ the relativistic effects might be important.

In the absence of the coupling to the $\pi \pi$ channel we can use similar relation between the $K \bar{K}$ scattering phase shift $\delta_{K \bar{K}}$ and the phase of the Jost function $D_{K}\left(k_{1}\right)$ as in Eq. (34) for the $\pi \pi$ channel. In the limit $k_{1} \longrightarrow 0$ the $K \bar{K}$ scattering length is given by $a_{K \bar{K}} \approx t g\left(\delta_{K \bar{K}}\right) / k_{1}$. The energy dependence of the phase shifts can be directly calculated using Eqs. (23), (25), (49) or (A13) for a specific choice of the bound state energy at a given value of the range parameter $\beta_{1}$. This is illustrated in Fig. 1a for two values of $\beta_{1}=150$ $\mathrm{MeV}$ and $2000 \mathrm{MeV}$. At the $K \bar{K}$ threshold the value of the phase shift is $\pi$ (conventionally assumed for a bound state) and monotonically decreases to zero as the energy increases. Again we see a very important difference between the relativistic and nonrelativistic expressions for large $\beta_{1}$ values $\left(\beta_{1}>m_{K}\right)$. At $\beta_{1}=2000 \mathrm{MeV}$ and $E=1400 \mathrm{MeV}$ the difference is as large as $30^{\circ}$. Let us also notice that at higher $\beta_{1}$ the phase shift decrease with energy is much less steep than at lower $\beta_{1}=150 \mathrm{MeV}$. This decrease at the $K \bar{K}$ threshold is governed by the negative value of the $K \bar{K}$ scattering length. The general expression for the $K \bar{K}$ scattering length in the relativistic case is given by Eq. (38) provided we substitute in it the $K \bar{K}$ channel parameters $m_{1}, \beta_{1}$ and $\Lambda_{11}$.

The values of the scattering lengths and the coupling constants are given

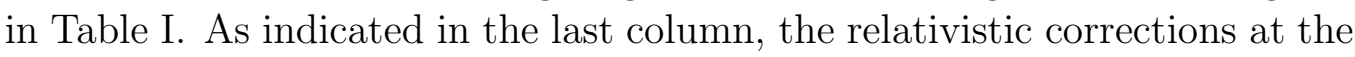


$K \bar{K}$ threshold amount to $13 \%$ if the value $\beta_{1}$ is as large as $2000 \mathrm{MeV}$, while at low $\beta_{1} \leq 150 \mathrm{MeV}$ they are smaller than $1 \%$.

Next we examine the main difference between the antibound state and the bound state discussed above. The corresponding Jost function zero is at $k_{1}=i \alpha_{1}$, but now $\alpha_{1}$ is negative. The nonrelativistic relation (43) leads to a weaker coupling than in the previous case $\left(-1<\Lambda_{11}<0\right)$. In Fig. 1b we show the energy dependence of the phase shifts calculated in the relativistic and nonrelativistic cases for two values of the range parameters $\beta_{1}$ as in Fig. 1a. The phase shifts increase at the $K \bar{K}$ threshold starting from the zero value, which means that the scattering length is positive. Again we notice that the relativistic effects are mainly important for the large values of the parameter $\beta_{1}$ and for higher energies. The phase shifts calculated relativistically are larger that the corresponding nonrelativistic values.

A special case in the $K \bar{K}$ channel is the existence of a resonance at the complex energy $M_{S}-i \Gamma_{S} / 2$, where $M_{S}$ is the resonance energy and $\Gamma_{S}$ is its width. In the complex momentum plane there are two $S$-matrix poles in the lower half plane at $k_{1}= \pm k_{R}-i k_{I}$, where $k_{I}>0$. They coincide with zeros of the Jost function:

$$
D_{K}\left( \pm k_{R}-i k_{I}\right)=0 .
$$

For our nonrelativistic and simple choice of the $K \bar{K}$ interaction (Eq. B) the interaction strength and the range parameter are fixed, since $\beta_{1}=k_{I}$ and $\Lambda_{11}=k_{R}^{2} / k_{I}^{2}>0$ (repulsive interaction). In the relativistic case, however, there are two possibilities of choosing the parameters $\beta_{1}$ and $\Lambda_{11}$ at a given resonance position. One set of parameters is very close to the nonrelativistic set. This is related to the values of $k_{I}$ which in general must be smaller than about $100 \mathrm{MeV}$ if we would try to attribute the discussed resonance to the observed rather narrow $f_{0}(975)$ meson strongly coupled to the $K \bar{K}$ channel. The second relativistic solution is obtained for very large $\beta_{1}$ value and $\Lambda_{11}<0$ (attractive interaction). The phase shifts are plotted in Fig. 1c and their behaviour is very different in the two cases. For smaller $\beta_{1}$ values close to $70 \mathrm{MeV}$ (upper curve) we at first observe an energy decrease and then an increase of the $\delta_{K \bar{K}}$ function. This means that the resulting scattering lengths are negative. The phase shifts calculated for the relativistic and nonrelativistic propagators are very similar. They differ by less than one degree so the corresponding lines in Fig. 1c are indistinguishable. The lower curve corresponds to the second relativistic solution and the phase shifts 
increase staring from $0^{\circ}$ at the threshold. In this case the scattering length is positive.

At the end of this section we return to the discussion of the bound state, especially its wave function in the momentum and configuration spaces. An important parameter describing the bound $K \bar{K}$ system near the threshold is the root mean square radius parameter. It has been discussed in the nonrelativistic model [18], and its preferable value was given as $\left\langle r_{S}\right\rangle^{1 / 2}=0.76$ $\mathrm{fm}$. The nonrelativistic form of the radial wave function is simple and can be found in Ref. [18]. Its Fourier transform $\Psi_{N}(p)$ describes the momentum distribution of the $K \bar{K}$ relative motion (see Ref. [41])

$$
\Psi_{N}(p)=c_{N} G_{N}(p) g_{1}(p),
$$

where $G_{N}(p)=-m_{K} /\left(\alpha_{1}^{2}+p^{2}\right)$ is the nonrelativistic propagator corresponding to the binding energy $E_{B}=\alpha_{1}^{2} / m_{K}$ and $c_{N}$ is the normalization constant

$$
c_{N}=-\frac{1}{2} \sqrt{\frac{\alpha_{1} \beta_{1}}{m_{K}}}\left(\alpha_{1}+\beta_{1}\right)^{3 / 2}
$$

such that $\int d^{3} k\left|\Psi_{N}(k)\right|^{2}=1$. Similarly in the relativistic case we write

$$
\Psi_{R}(p)=c_{R} G_{R}(p) g_{1}(p)
$$

where $G_{R}(p)=1 /\left(E-2 \sqrt{p^{2}+m_{K}^{2}}\right)$ is the relativistic propagator corresponding to the total energy $E=2 \sqrt{m_{K}^{2}-\alpha_{1}^{2}}$ and $c_{R}$ is the normalization constant. The relativistic wave function in the configuration space $\Phi_{R}(r)$ is related to $\Psi_{R}(p)$ by the Fourier transform. An important parameter describing the radial extension of the $K \bar{K}$ system is the root mean square diameter

$$
\left\langle r^{2}\right\rangle=\int d^{3} r r^{2}\left|\Phi_{R}(r)\right|^{2}
$$

or the root mean square radius $\left\langle r_{S}^{2}\right\rangle^{1 / 2}=\frac{1}{2}\left\langle r^{2}\right\rangle^{1 / 2}$. The results of numerical calculations are given in Table [1]. The first set of parameters $\alpha_{1}$ and $\beta_{1}$ is taken from Ref. [18]. The second and third line correspond to the results of fits described further in Sect. V. The root mean square radii are small, typically about $0.7 \mathrm{fm}$, so the $K \bar{K}$ molecule has a rather compact structure. This fact can have important consequences. For example, in Ref. [25] a value 
$1.2 \mathrm{fm}$ has been used to predict the width of the radiative $\phi \rightarrow f_{0}(975) \gamma$ decay. If instead of $1.2 \mathrm{fm}$ we use the value $0.7 \mathrm{fm}$ the corresponding width increases by a factor of 2 . Let us mention that the annihilation probability of the $K \bar{K}$ molecule into two photons will be substantially increased for small $K \bar{K}$ radii (compare 42]). In Table II we can also notice a general trend: the root mean square radii calculated for the relativistic propagator are smaller by about $0.1 \mathrm{fm}$ in comparison with corresponding values for the nonrelativistic case. The difference comes from the behaviour of the radial wave functions for the values of $r$ smaller than about $0.7 \mathrm{fm}$, where the relativistic wave function takes higher values than the nonrelativistic one.

\section{V $\pi \pi$ and $K \bar{K}$ coupled channel analysis}

In this section we describe the coupled channel analysis performed for the interacting $\pi \pi$ and $K \bar{K}$ pairs. Starting from the $\pi \pi$ threshold we put the upper energy limit at $1400 \mathrm{MeV}$, where one can still neglect the coupling to other channels with higher thresholds (as discussed in [19]). The interactions (3 5) in both channels have the forms as simple as possible. As it was mentioned in Sect. III the rank-two potential allows us to describe a substantial increase of the $\pi \pi$ phase shifts below $1 \mathrm{GeV}$ and the existence of the known $f_{0}(1400)$ meson. All the potential parameters have been obtained by fitting the calculated phase shifts $\delta_{\pi \pi}, \delta_{K \bar{K}}$ and the inelasticity parameter $\eta$ to the experimental data. Above the $K \bar{K}$ threshold a sum $\varphi=\delta_{\pi \pi}+\delta_{K \bar{K}}$ is often used and a quantity $x=\left(1-\eta^{2}\right) / 4$ is introduced to represent the inelasticity. Except for the $\pi \pi$ threshold data of Refs. [28], [29] we have used the data of Refs. 30], [31] covering a wide energy band. The total number of the $\delta_{\pi \pi}$ points is 56. In addition, 17 points of the $\eta$ dependence have been taken from Ref. [43]. For the $\varphi$ dependence we use two distinct data sets [43, 444 which essentially differ in their behaviour near the $K \bar{K}$ threshold. In the data 45] used in the analysis of Ref. 44 a constant increase of $\varphi$ is seen while in the data of Ref. [43] some threshold decrease with energy can be observed. As it was shown in Sect. IV, this difference could be crucial for understanding the nature of $f_{0}(975)$, so we have performed two separate fits to the collection of the $\delta_{\pi \pi}, \delta_{K \bar{K}}$ and $\eta$ points. The data set containing $16 \varphi$-points of Ref. 433. is further called set 1 while in the set 2 we include $17 \varphi$-numbers read from Ref. [44] (the total number of the data points is 89 and 90 respectively for 
these two data sets).

The fitted parameters are shown in Table III. The asymmetric errors $\Delta_{+}$ and $\Delta_{-}$correspond to an increase of the total $\chi^{2}$ value by one unit. Let us notice that all the channel coupling constants $\Lambda_{i i}$ are negative, which means that the interactions are attractive in both channels. The couplings between channels are small. The ranges of the form factors are short since the $\beta_{i}$ values are in the $\mathrm{GeV}$ region. During the fitting procedure we have found that it was convenient to use as an independent variable the product $\Lambda_{33} \beta_{3}$ instead of $\beta_{3}$. For both data sets this product is very close to $-\pi m_{\pi} \simeq-431$ $\mathrm{MeV}$. This fact is not accidental as it is explained in Appendix. The very large value of $\beta_{3}$ is compensated by very small values of the corresponding coupling constants $\Lambda_{33}$ and $\Lambda_{13}$. The starting parameters $\Lambda_{22}$ and $\beta_{2}$ in the minimization procedure have been given in Sect. III. Other coupling constants were initially put equal to zero and $\beta_{i}$ parameters had the value $1 \mathrm{GeV}$. In Table $\mathbb{\square}$ we show $\chi^{2}$ values of two fits for separated data parts. The data on $\delta_{\pi \pi}$ and $\eta$ are very well described by both fits but the data on $\varphi$ only by the fit to the data set 1 .

In figures 2-4 the results of our fits to the data sets 1 and 2 are shown. Theoretical $\delta_{\pi \pi}$ and $\eta$ values differ only slightly for both fits. In Fig. 3 we notice an initial decrease of the $\varphi$ curves at the $K \bar{K}$ threshold, so the data set 1 is clearly favoured by the model (compare also the corresponding $\chi^{2}$ values of Table [V]. Due to a very similar monotonic increase of the $\delta_{\pi \pi}$ phase shifts above $1 \mathrm{GeV}$, the differences in the $\varphi$ behaviour for the data sets 1 and 2 are related to various possible trends of the $\delta_{K \bar{K}}$. As discussed in Sect. IV the trend seen in the $\varphi$-data of Ref. [43] is connected with a presence of the quasibound $K \bar{K}$ state but in order to exclude other possibilities new precise $\delta_{K \bar{K}}$ measurements near the threshold are needed.

We have investigated a pole structure of the $S$ matrix elements and the results are shown in Table $\mathrm{V}$. A very wide $f_{0}(500)$ meson (see Sect. III) causes a systematic increase of the $\delta_{\pi \pi}$ phase shifts starting from the $\pi \pi$ threshold. The presence of the narrow $f_{0}(975)$ manifests itself by a strong jump of the $\delta_{\pi \pi}$ and the enhancement of the inelasticity function $x$ near the $K \bar{K}$ threshold (see Figs. 2 and 4). A further increase of the $\delta_{\pi \pi}$ and $\varphi$ above $1.2 \mathrm{GeV}$ is related to the presence of the $f_{0}(1400)$ meson. Also the structure of the inelasticity around the energy $1.3 \mathrm{GeV}$ is caused by this state. We should point out that the values of the resonance parameters may be changed if a fit to another data sets is performed. We expect that changes 
may be much larger than the error ranges quoted in Table $\nabla$. This is due to the fact that some experiments on the $\pi \pi$ and $K \bar{K}$ scattering have supplied contradictory results, as was illustrated by our choice of the sets 1 and 2 . We think, however, that the differences between data sets are representative. In particular the resonance parameters are not very much influenced if we compare results of two fits in Table $\mathrm{\nabla}$. Let us mention here that a moderate width of the $f_{0}(1400)$ is obtained together with the presence of the wide $f_{0}(500)$ meson. The calculated masses and widths of the $f_{0}(1400)$ meson are in very good agreement with the corresponding values obtained in ref. [43] $(M=1425 \pm 15 \mathrm{MeV}$ and $\Gamma=160 \pm 30 \mathrm{MeV})$. In the absence of the former state the systematic increase of the $\pi \pi$ phase shifts below the energy of $1 \mathrm{GeV}$ should be caused by a very wide $f_{0}(1400)$ state (see for example Ref. [18]) or by another wide state at the energy of about $1 \mathrm{GeV}$.

In Fig. 5 the $S_{\pi \pi}$ matrix element singularities are presented using the complex variable $z$ (compare [46] and [18])

$$
z=\frac{k_{1}+k_{2}}{\sqrt{m_{K}^{2}-m_{\pi}^{2}}} .
$$

The positions of poles and zeros have been calculated from the fit to the data set 1 . A very similar structure of the $S_{\pi \pi}$ has been found for the fit to the data set 2 . The energy sheets on the $z$-plane are defined by the signs of the imaginary parts of the $k_{2}$ and $k_{1}$ momenta $\left(\operatorname{Im} k_{2}, \operatorname{Im} k_{1}\right)$ as follows: I $(+,+)$, II $(-,+)$, III $(-,-)$, IV $(+,-)$. In Fig. 5 we notice two poles on sheet II labelled as 1 and 2 . They correspond to the $f_{0}(500)$ and $f_{0}(975)$ resonances. The $f_{0}(1400)$ resonance position on sheet III is indicated by 3 . Those poles are the nearest singularities lying close to the physical region. The $\pi \pi$ threshold region is also strongly influenced by the cuts located on the imaginary $z$ axes. In the momentum space these cuts are on the $k_{i}$ axes (from $\pm i m_{i}$ to $\pm i \infty)$. Their origin is due to the presence of the logarithmic and square root functions in the $J_{i j}$ integrals (see Appendix). In the nonrelativistic case these integrals have much simpler structure and there are no cuts in the $z-$ plane. Two poles at $\operatorname{Re} z>0$ are particularly close to the $|z|=1$ circle: pole 2 associated with the $f_{0}(975)$ resonance and the another pole lying on sheet III with a zero almost superposed on its top. Pole 2 can be related to the antibound state discussed in Sect. IV. If the coupling between channels is switched off then the poles move to the $|z|=1$ circle. The positions of 
other zeros or poles are connected with the discussed $f_{0}(500)$ and $f_{0}(1400)$ resonances. In particular the structure of the inelasticity function shown in Fig. 4 is a result of an interplay of the $S$-matrix zeros and poles. The $S_{\pi \pi}$ matrix element also has two second order zeros related to the form of the $\pi \pi$ potential. They correspond to the values of $k_{2}=-i \beta_{2}$ and $k_{2}=-i \beta_{3}$ and due to large values of $\beta_{2}$ and $\beta_{3}$ two of them lay very close to the origin of the $z$-plane and the other two very far from that point. These poles are not shown in Fig. 5.

We have also performed fits with only one term in the $\pi \pi$ potential i.e. with $\lambda_{33}=0$ in Eq. (4). The $\chi^{2}$ fit was poor. In this case the $S_{\pi \pi}$ matrix element has no pole corresponding to the $f_{0}(1400)$ meson and the $\delta_{\pi \pi}$ and $\varphi$ phase shifts have a very flat behaviour above the energy $1.2 \mathrm{GeV}$.

Similar fits as for the relativistic form have been done using a nonrelativistic form of the Jost function. The coupling constants $\Lambda_{22}$ and $\Lambda_{33}$ had then opposite signs and the $f_{0}(975)$ meson also appeared as a $K \bar{K}$ bound state. We have found that apart from the $f_{0}(975)$ meson there was only one very wide $(\Gamma \approx 1300 \mathrm{MeV})$ state at the energy of about $850 \mathrm{MeV}$. The $\chi^{2}$ value for the fit was 283 for 89 points so it was much worse than in the relativistic case. Energy dependence of the $\delta_{\pi \pi}, \varphi$ and $x$ was similar to that one in the relativistic fit with only one term in the $\pi \pi$ potential. Like in Ref. [18], the $S$ matrix also has two poles on the negative part of the imaginary axis $k_{2}$ but no poles corresponding to the $f_{0}(500)$.

Scattering lengths $a_{\pi \pi}$ and $a_{K \bar{K}}$ are very important quantities describing the near threshold $\pi \pi$ and $K \bar{K}$ interactions. Comparison of the values obtained by us with experimental measurements and other theoretical estimations is shown in Table V1. Good agreement of the predicted $a_{\pi \pi}$ values for the relativistic case with other data is found. In the nonrelativistic case there are two $S$-matrix poles on the $k_{2}$ imaginary axis and one of them is relatively close to the $\pi \pi$ threshold, which gives a large positive contribution to the scattering length. Therefore the scattering length is much larger than in the relativistic case. An appearance of the imaginary part of the $K \bar{K}$ scattering length is related to the fact that above the $K \bar{K}$ threshold the annihilation process into two pions is possible. The value of this imaginary part is evaluated using a low momentum approximation of the inelasticity $\eta$

$$
\eta \approx 1-2 b k_{1}+O\left(k_{1}^{2}\right)
$$

where the parameter $b$ is equal to $\operatorname{Im} a_{K \bar{K}}$ (see also Ref. [19]). The negative 
value of the real part of the $K \bar{K}$ scattering length is caused by an influence of a single pole lying close to the $K \bar{K}$ threshold on the sheet II (see Fig. 5). This pole is related with the narrow $f_{0}(975)$ meson. We expect that in a case when two poles are close to the $K \bar{K}$ threshold on sheets II and III (as discussed in Ref. [17]) the absolute value of the real part of the $K \bar{K}$ scattering length can be much smaller. The reason is that the contributions of such poles have opposite signs so they cancel to a large extend. The cancellation is complete if the poles lie symmetrically in the kaon momentum space (at $k_{1}$ and $-k_{1}$ ). The precise experimental measurements of this quantity could distinguish between these two possibilities. There is one experimental estimation of the $K \bar{K}$ scattering length based on the data of ref. 477 which gives numbers similar to our findings. Unfortunately, the $K \bar{K}$ phase shifts used by Wetzel et al. do not satisfy a very general requirement $\delta_{K \bar{K}}\left(-k_{1}\right)=-\delta_{K \bar{K}}\left(k_{1}\right)$, so the values of the scattering lengths obtained in [47] could be questioned. The $K^{+} K^{-}$scattering lengths quoted in ref. 48 and given in Table V1 are theoretical estimations used in the calculations of the properties specific to the kaonic atoms. Contrary to the $K \bar{K}$ case, more information on the $\pi \pi$ scattering length is available. Our values for the relativistic fits are in good agreement with other determinations (see Table VI).

Due to the channel coupling the $f_{0}(975)$ and $f_{0}(1400)$ mesons can decay into the $\pi \pi$ and $K \bar{K}$ pairs. The $f_{0}(975)$ meson being presumably a $K \bar{K}$ bound state becomes unstable due to the annihilation process $K \bar{K} \rightarrow \pi \pi$. The branching ratio of its decay into the $K \bar{K}$ channel is

$$
B=\frac{\Gamma_{K} \bar{K}}{\Gamma_{t o t}},
$$

where $\Gamma_{\text {tot }}$ denotes the total and $\Gamma_{K \bar{K}}$ the $K \bar{K}$ fractional decay width. If we wish to compare the experimental $f_{0}(975)$ branching ratio with the theoretical one, we must take into account the fact that due to the proximity of the $K \bar{K}$ threshold the $f_{0}(975)$ resonance has no a typical Breit-Wigner shape. As it was discussed in Ref. [19] the average branching ratio $B_{a v}$ of the $f_{0}(975)$ reads

$$
B_{a v}=\frac{1}{2\left(M_{\max }-M_{s}\right)} \int_{M_{t h}}^{M_{\max }} d E\left(1-\eta^{2}\right),
$$

where $M_{\max }, M_{s}$ and $M_{t h}$ denote respectively the upper limit of integration, mass of the meson and mass of the $K \bar{K}$ threshold. In the numerical calculations we put $M_{\max }=4 \Gamma_{t o t}$. We obtain $B_{a v}=18.0 \pm 0.7 \%$ for the fit 
to the data set 1 and $B_{a v}=18.4 \pm 0.6 \%$ for the fit to the data set 2 in agreement with the Particle Data Group value $21.9 \pm 2.4 \%$ 34. In the case of the $f_{0}(1400)$ meson we have performed the integration from $M_{s}-\Gamma_{t o t}$ to $M_{s}+\Gamma_{t o t}$ substituting $2 \Gamma_{t o t}$ in place of $2\left(M_{\max }-M_{s}\right)$ in Eq. (52). For the $f_{0}(1400) \rightarrow K \bar{K}$ branching ratio we obtain $16 \pm 1 \%$ for both data sets in agreement with [43]. Let us notice that the $f_{0}(1400)$ resonance mass is slightly higher than the upper energy limit $1.4 \mathrm{GeV}$ used in the fits. This fact may lead to some uncertainties in the predicted values of the $f_{0}(1400)$ branching ratios. The Particle Data Group value is $6.4_{-2.5}^{+1.9 \%}$ [34]. It is entirely based on the analysis done in Ref. [49] in the energy range from 1100 to $1420 \mathrm{MeV}$. The corresponding value of the branching ratio calculated for the parameter set 1 in the same energy range is as high as $30.3 \%$. This is a result of the existing discrepancy between the $s$-wave intensities obtained by the Argonne group 43] and the CERN-Cracow-Munich group [49]. In our analysis we have chosen the Argonne data, since they include points closer to the $K \bar{K}$ threshold. For energies higher than $1.4 \mathrm{GeV}$ an influence of other scalarisoscalar states such as $f_{0}(1525), f_{0}(1590)$ or even $f_{0}(1710)$ (see references in [34]) may be important. We have also tried to include in our analysis the energy range from $1.4 \mathrm{GeV}$ to $1.5 \mathrm{GeV}$ and we could not simultaneously fit the $\delta_{\pi \pi}$ and $\varphi$ phase shifts. The $\delta_{\pi \pi}$ data in this energy region favour wider

$f_{0}(1400)$ meson; the sum $\varphi=\delta_{\pi \pi}+\delta_{K \bar{K}}$ which increases steeply with energy requires an existence of a narrow state.

\section{Conclusions}

We have performed the analysis of the isoscalar spin zero $\pi \pi$ and $K \bar{K}$ coupled channel interactions. The model was based on a separable potential formalism. Two channel scattering amplitudes have been evaluated from the coupled equations of the Lippmann-Schwinger type. Using the relativistic propagators we have obtained very good fits to the experimental $\pi \pi$ and $K \bar{K}$ scattering data. It has been found that the potentials in the $\pi \pi$ and $K \bar{K}$ channels are attractive and have short range form factors. The $\chi^{2}$ fits in the relativistic case were 3 or 4 times better than in the nonrelativistic case. In contrast to the nonrelativistic calculations (see [18] and Sect. V) new $f_{0}(500)$ state has been found in the $\pi \pi$ interactions treated relativistically. Its large width about $500 \mathrm{MeV}$ is in agreement with other estimations. In the 
relativistic version of our model the mass and width of the $f_{0}(1400)$ meson are well described (see Table $\mathrm{V}$ ). Relativistic effects also play the important role near the $\pi \pi$ threshold. The $\pi \pi$ scattering length is significantly smaller than in the nonrelativistic case and is in agreement with the experimental data and other models (see Table $\mathrm{VI}$ ).

We have also been interested in describing the $K \bar{K}$ interaction. Two different data sets on the $K \bar{K}$ phase shifts have been taken into account. Our solutions indicate the existence of a quasibound state below the $K \bar{K}$ threshold which we identify with the $f_{0}(975)$ meson. Thus this state does not seem to be a typical $q \bar{q}$ meson. Some observables, e.g. the $K \bar{K}$ scattering length and root mean square radius of the $f_{0}(975)$ have been evaluated. We predict the large negative value of the $K \bar{K}$ scattering length about $-1.7 \mathrm{fm}$ and the corresponding imaginary part of the order of $0.6 \mathrm{fm}$ (Table VI). In our calculations the $f_{0}(975)$ state appears as the compact $K \bar{K}$ system with the root mean square radius of about $0.7 \mathrm{fm}$, so it is not a $K \bar{K}$ molecule of the deuteron size. Analysis of the $K \bar{K}$ channel leads to a conclusion that at the $K \bar{K}$ threshold the relativistic corrections to the $K \bar{K}$ scattering length or the root mean square radius are of the order of $10 \%$ and they gradually grow with increasing energy.

The existing data above the $K \bar{K}$ threshold are still controversial. Comparison of our expectations with new experimental data may be done in near future and a new light on the nature of the $f_{0}(975)$ and $a_{0}(980)$ mesons can be shed on if very precise measurements near the $K \bar{K}$ production threshold like those planned at COSY [50] are performed. Very good energy resolution would enable us to take into account a mass splitting between the charged $K^{-} K^{+}$and neutral $K^{0} \overline{K^{0}}$ modes. Due to different interactions of the $K^{-}$ and $K^{+}$with nucleons in the GeV region the molecular picture of the $f_{0}(975)$ can also be verified by studying its interaction inside nuclear matter (see also Ref. [19).

\section{Acknowledgments}

This work has been partially supported by Polish Committee for Scientific Research (grant No 20198 9101). Discussions with L. Görlich, A. D. Martin, M. Różańska, K. Rybicki and J. Turnau are gratefully acknowledged. We thank very much D. Morgan for his computer communications. 


\section{Appendix}

In this appendix we give explicit expressions for the $t$-matrix elements (11) in terms of the coupling constants $\lambda_{i j}$ and the integrals $I_{i j}$ (Eqs. (14) and $(15)$ ):

$$
\begin{aligned}
t_{11}= & D^{-1}\left[\lambda_{11}-\left(\lambda_{11} \lambda_{22}-\lambda_{12}^{2}\right) I_{22}-\left(\lambda_{11} \lambda_{33}-\lambda_{13}^{2}\right) I_{33}+2 \lambda_{12} \lambda_{13} I_{23}\right. \\
+ & \left.\left(\lambda_{11} \lambda_{22} \lambda_{33}-\lambda_{33} \lambda_{12}^{2}-\lambda_{22} \lambda_{13}^{2}\right)\left(I_{22} I_{33}-I_{23}^{2}\right)\right] \\
t_{12}= & D^{-1}\left(\lambda_{12}-\lambda_{12} \lambda_{33} I_{33}+\lambda_{22} \lambda_{13} I_{23}\right) \\
t_{13}= & D^{-1}\left(\lambda_{13}-\lambda_{13} \lambda_{22} I_{22}+\lambda_{33} \lambda_{12} I_{23}\right) \\
t_{22}= & D^{-1}\left\{\lambda_{12} I_{11}\left[\lambda_{12}\left(1-\lambda_{33} I_{33}\right)+\lambda_{22} \lambda_{13} I_{23}\right]\right. \\
+ & \left.\lambda_{22}\left(1-\lambda_{11} I_{11}\right)\left(1-\lambda_{33} I_{33}\right)-\lambda_{22} \lambda_{13} I_{11}\left(\lambda_{12} I_{23}+\lambda_{13} I_{33}\right)\right\} \\
t_{23}= & D^{-1}\left[\lambda_{22} \lambda_{33} I_{23}+\lambda_{12} \lambda_{13} I_{11}\right. \\
& \left.-\left(\lambda_{11} \lambda_{22} \lambda_{33}-\lambda_{22} \lambda_{13}^{2}-\lambda_{33} \lambda_{12}^{2}\right) I_{11} I_{23}\right] \\
t_{33}= & D^{-1}\left\{\lambda_{13} I_{11}\left[\lambda_{13}\left(1-\lambda_{22} I_{22}\right)+\lambda_{33} \lambda_{12} I_{23}\right]\right. \\
+ & \left.\lambda_{33}\left(1-\lambda_{11} I_{11}\right)\left(1-\lambda_{22} I_{22}\right)-\lambda_{33} \lambda_{12} I_{11}\left(\lambda_{13} I_{23}+\lambda_{12} I_{22}\right)\right\}
\end{aligned}
$$

In Eqs. (A1)-(A6) $D$ denotes the function defined in Eq. (24). The integrals ( 14) and ( 15) have also been used in 23] to calculate the $K^{-}-N$ potential but analytical formulas have been given only when the propagator $G$ in Eq. (2) was nonrelativistic (see also [51]). We show below that in the relativistic case the analytical calculations can be performed as well. A very helpful substitution is: $y=\sqrt{s^{2} /\left(s^{2}+m_{i}^{2}\right)}$. Then after some straightforward algebraic operations the integrals $I_{i j}$ can be rewritten in the terms of the integral

$$
F\left(x^{2}\right)=\int_{0}^{1} \frac{d y}{1-y^{2} x^{2}+i \epsilon} .
$$

If $x^{2}$ is real then

$$
F\left(x^{2}\right)= \begin{cases}\frac{1}{2 x} \ln \left(\frac{x+1}{x-1}\right)-\frac{i \pi}{2 x} & \text { if } x^{2}>1 \\ \frac{1}{2 x} \ln \left(\frac{1+x}{1-x}\right) & \text { if } 0<x^{2}<1 \\ 1 & \text { if } x^{2}=0 \\ \arctan (|x|) /|x| & \text { if } x^{2}<0\end{cases}
$$


Finally the integrals $I_{i j}$ can be expressed in terms of $J_{i j}$ (Eq. (19)):

$$
\begin{gathered}
J_{i i}\left(k_{i}\right)=-\frac{E_{i}}{4 m_{i}\left(1-i a_{i}\right)^{2}}-\frac{2}{\pi b_{i}\left(1+a_{i}^{2}\right)^{2}} \\
\times\left\{\frac{1}{2}\left(1+a_{i}^{2}\right)+\left[-\frac{1}{2}\left(1+a_{i}^{2}\right) b_{i}^{2}+a_{i}^{2} C_{i}^{2}\right] d_{i}-a_{i}^{2} C_{i}^{2} H_{i}\right\}, \\
J_{23}\left(k_{2}\right)=-\frac{E_{2}}{2 m_{2}\left(1-i a_{2}\right)\left(1-i a_{3}\right)} \frac{\sqrt{\beta_{2} \beta_{3}}}{\beta_{2}+\beta_{3}}+\frac{2}{\pi \sqrt{b_{2} b_{3}}\left(1+a_{2}^{2}\right)\left(1+a_{3}^{2}\right)} \\
\times\left\{\frac{E_{2}^{2}}{4 \beta_{2} \beta_{3}} H_{2}+\frac{\beta_{2} \beta_{3}}{\beta_{3}^{2}-\beta_{2}^{2}}\left[d_{3}\left(1+a_{2}^{2}\right)\left(b_{3}^{2}-1\right)-d_{2}\left(1+a_{3}^{2}\right)\left(b_{2}^{2}-1\right)\right]\right\} .
\end{gathered}
$$

In $($ A9 $)$ and $($ A10 $)$

$$
E_{i}=2 \sqrt{k_{i}^{2}+m_{i}^{2}}, C_{i}=\frac{E_{i}}{2 k_{i}}, H_{i} \equiv F\left(C_{i}^{2}\right)
$$

and

$$
a_{i}=\frac{k_{i}}{\beta_{i}}, b_{i}=\frac{m_{i}}{\beta_{i}}, d_{i} \equiv F\left(1-b_{i}^{2}\right), \quad i=1,2,3 .
$$

In (A11) and (A12) the $m_{i}$ are defined by (7) and $k_{3} \equiv k_{2}$.

The nonrelativistic limit of the integrals (14) and (15) can be obtained from (A9) and (A10) when $m_{i} \longrightarrow \infty$. Physically it means that the nonrelativistic limit is achieved when both $k_{i}(i=1,2)$ and $\beta_{j}(j=1,2,3)$ are much smaller than $m_{i}$. Then

$$
J_{i i}\left(k_{i}\right)=-\frac{1}{\left(1-i a_{i}\right)^{2}}
$$

and

$$
J_{23}\left(k_{2}\right)=-\frac{2 \sqrt{\beta_{2} \beta_{3}}}{\left(\beta_{2}+\beta_{3}\right)\left(1-i a_{2}\right)\left(1-i a_{3}\right)} .
$$

Let us discuss the limit $\beta_{3} \longrightarrow \infty$. For very large $\beta_{3}$ the integral $J_{33}$ is proportional to $\beta_{3}$ :

$$
J_{33} \simeq-\frac{\beta_{3}}{\pi m_{\pi}}
$$

In order to compensate too large values of $J_{33}$ in Eq. 26-28) we can multiply it by a sufficiently small $\Lambda_{33}$ value which should be inversely proportional to 
$\beta_{3}$. If $\beta_{3} \longrightarrow \infty$ and $\Lambda_{33} \beta_{3}=-\pi m_{\pi}$ then the term $1-\Lambda_{33} J_{33}$ in Eq. (26) does not grow with $\beta_{3}$.

In the limit $\beta_{3} \longrightarrow \infty$ the integral $J_{23}$ vanishes in Eq. (28) and the Jost function $D_{\pi}\left(k_{2}\right)$ is approximately a product of two terms $\left(1-\Lambda_{22} J_{22}\right)\left(1-\Lambda_{33} J_{33}\right)$. The zero of the first term can be attributed to the $f_{0}(500)$ meson as discussed in Sect. III. For the values $\beta_{3}$ and $\Lambda_{33}$ as given in Table III the second term (together with the small $J_{23}^{2}$ term) allows us to obtain the second $D_{\pi}$ zero relatively close to the real axis $k_{2}$. In Table we assign this zero to the $f_{0}(1400)$ meson.

\section{References}

[1] T. H. Burnett and S. R. Sharpe, Annu. Rev. Nucl. Part. Sci. 40, 327 (1990).

[2] N. Isgur and K. Königsmann, Nucl. Phys. A527, 491c (1991).

[3] R. Landua, in Proceedings of the Joint Intern. Lepton-Photon Symp. and Europhysics Conf. on High Energy Physics (Geneva 1991), edited by S. Hegarty, K. Potter and E. Quercigh, (World Scientific, Singapore, 1992), Vol. 2, p. 377.

[4] G. Karl, Universität München Report No. LMU-22/92.

[5] R. J. Jaffe, Phys. Rev. D 15, 267 (1977).

[6] N. N. Achasov, S. A. Devyanin and G. N. Shestakov, Usp. Fiz. Nauk 142, 361 (1984).

[7] J. Weinstein and N. Isgur, Phys. Rev. D 27, 588 (1983).

[8] T. Barnes, Phys. Lett. 165B, 434 (1985).

[9] N. A. Törnqvist, Phys. Rev. Lett. 67, 556 (1991).

[10] A. B. Wicklund et al., Phys. Rev. Lett. 45, 1469 (1980).

[11] S. Wycech and A. M. Green, Nucl. Phys. A562, 446 (1993).

[12] S. J. Lindenbaum, Report No. BNL-47885 (1992). 
[13] C. A. Heusch, Nucl. Phys. (Proc. Suppl.) B27, 263 (1992).

[14] N. A. Törnqvist, Phys. Rev. Lett. 49, 624 (1982).

[15] N. N. Achasov and G. N. Shestakov, Usp. Fiz. Nauk 161, 53 (1991).

[16] D. Morgan and M. R. Pennington, Phys. Lett. 258B, 444 (1991) [erratum in Phys. Lett. 269B, 477 (1991)].

[17] D. Morgan and M. R. Pennington, Phys. Rev. D 48, 1185 (1993).

[18] F. Cannata, J.-P. Dedonder and L. Leśniak, Z. Phys. A 334, 457 (1989).

[19] F. Cannata, J.-P. Dedonder and L. Leśniak, Z. Phys. A 343, 451 (1992).

[20] J. Weinstein and N. Isgur, Phys. Rev. D 41, 2236 (1990).

[21] D. Lohse et al., Nucl. Phys. A516, 513 (1990).

[22] J. T. Londergan, K. W. McVoy and E. J. Moniz, Ann. Phys. 86, 147 (1974).

[23] P. B. Siegel and W. Weise, Phys. Rev. C 38, 2221 (1988).

[24] P. J. Fink, Jr. et al., Phys. Rev. C 41, 2720 (1990).

[25] F. E. Close, N. Isgur and S. Kumano, Nucl. Phys. B389, 513 (1993).

[26] Z. P. Li, F. E. Close and T. Barnes, Phys. Rev. D 43, 2161 (1991).

[27] Y. Yamaguchi and Y. Yamaguchi, Phys. Rev. 95, 1635 (1954).

[28] L. Rosselet et al., Phys. Rev. D 15, 574 (1977).

[29] A. A. Belkov et al., Pisma Zh. Eksp. Teor. Fiz. 29, 652 (1979).

[30] V. Srinivasan et al., Phys. Rev. D 12, 681 (1975).

[31] G. Grayer et al., Nucl. Phys. B75, 189 (1974).

[32] J. Lowe et al., Phys. Rev. C44, 956 (1991).

[33] H. Burkhardt and J. Lowe, Phys. Rev. Lett. 67, 2622 (1991). 
[34] Particle Data Group, Phys. Rev. D 45, part II (1992).

[35] G. V. Efimov, M. A. Ivanov and S. G. Mashnik, Dubna Report JINR-E2-89-780 (1989).

[36] B. R. Martin, D. Morgan and G. Shaw, Pion-Pion Interactions in Particle Physics (Academic Press Inc. London (1976)), pp. 296-299.

[37] R. Machleidt, K. Holinde and Ch. Elster, Phys. Rep. 149, 1 (1987).

[38] K. Nakayama and S. Krewald, Phys. Lett. 273B, 199 (1991).

[39] Particle Data Group, Phys. Lett. 50B, 1 (1974).

[40] R. G. Newton, Scattering Theory of Waves and Particles (McGraw-Hill Book Company, N.York 1966) p. 311n.

[41] J. M. Eisenberg and W. Greiner, Microscopic theory of the nucleus (North-Holland Publishing Company, Amsterdam, 1976) p. 67.

[42] T. Barnes, Phys. Lett. 165B, 434 (1985).

[43] D. Cohen et al., Phys. Rev. D 22, 2595 (1980).

[44] P. Estabrooks, Phys. Rev. D 19, 2678 (1979).

[45] A. J. Pawlicki et al., Phys. Rev. D 15, 3196 (1977).

[46] M. Kato, Ann. Phys. (N.Y) 31, 130 (1965).

[47] W. Wetzel et al., Nucl. Phys. B115, 208 (1976).

[48] O. Dumbrajs, Z. Phys. A 321, 297 (1985).

[49] L. Görlich et al., Nucl. Phys. B174, 16 (1980).

[50] W. Oelert, in Proceedings of the Workshop on Meson Production, Interaction and Decay, Cracow, 1991, edited by A. Magiera, W. Oelert and E. Grosse (World Scientific, London, 1991), p. 199; M. Kistryn et al., ibid. p. 217.

[51] M. Alberg, E. M. Henley and L. Wilets, Ann. Phys. (N.Y) 96, 43 (1976). 
Table I: $K \bar{K}$ coupling constants $\Lambda_{11}$ and scattering lengths $a$ corresponding to the $K \bar{K}$ bound state at $975 \mathrm{MeV}$ (without coupling to the $\pi \pi$ channel).

\begin{tabular}{|c|c|c|c|c|c|}
\hline \multirow{2}{*}{$\begin{array}{c}\beta_{1} \\
(\mathrm{MeV})\end{array}$} & \multicolumn{2}{|c|}{ nonrelativistic } & \multicolumn{2}{|c|}{ relativistic } & \multirow[b]{2}{*}{$\left|\frac{a_{R}}{a_{N}}-1\right| \cdot 100 \%$} \\
\hline & $\Lambda_{11}$ & $a_{N}(\mathrm{fm})$ & $\Lambda_{11}$ & $a_{R}(\mathrm{fm})$ & \\
\hline 150 & -2.55 & -4.32 & -2.48 & -4.28 & 1 \\
\hline 500 & -1.39 & -2.81 & -1.18 & -2.70 & 4 \\
\hline 2000 & -1.09 & -2.35 & -0.54 & -2.05 & 13 \\
\hline
\end{tabular}

Table II: Root mean square radii of the $K \bar{K}$ wave function.

\begin{tabular}{|c|c|c|c|c|}
\hline$E$ & $\alpha_{1}$ & $\beta_{1}$ & $\begin{array}{c}\text { nonrelativistic } \\
\left\langle r_{S}^{2}\right\rangle^{1 / 2}\end{array}$ & $\begin{array}{c}\text { relativistic } \\
\left\langle r_{S}^{2}\right\rangle^{1 / 2}\end{array}$ \\
$\mathrm{MeV}$ & $\mathrm{MeV}$ & $\mathrm{MeV}$ & $\mathrm{fm}$ & $\mathrm{fm}$ \\
\hline 971.65 & 98.90 & 2000 & 0.76 & 0.66 \\
973.36 & 94.51 & 1496 & 0.81 & 0.73 \\
973.71 & 93.59 & 2177 & 0.80 & 0.69 \\
\hline
\end{tabular}


Table III: Model parameters and their up $\left(\Delta_{+}\right)$and down $\left(\Delta_{-}\right)$errors.

\begin{tabular}{|l|r|r|r|r|r|r|}
\hline \multicolumn{1}{c|}{ fitted } & \multicolumn{3}{|c|}{ set 1} & \multicolumn{1}{c|}{ set 2} \\
\cline { 2 - 7 } \multicolumn{1}{c|}{ parameters } & \multicolumn{1}{|c|}{ values } & \multicolumn{1}{c|}{$\Delta_{+}$} & \multicolumn{1}{c|}{$\Delta_{-}$} & \multicolumn{1}{c|}{ values } & \multicolumn{1}{c|}{$\Delta_{+}$} & \multicolumn{1}{c|}{$\Delta_{-}$} \\
\hline$\Lambda_{11}$ & -0.658 & 0.030 & -0.031 & -0.511 & 0.075 & -0.078 \\
$\Lambda_{22}$ & -0.201 & 0.003 & -0.003 & -0.201 & 0.004 & -0.004 \\
$\Lambda_{33} \times 10^{5}$ & -7.46 & 2.32 & -3.51 & -8.95 & 3.11 & -5.55 \\
$\Lambda_{12}$ & 0.0363 & 0.0021 & -0.0025 & 0.0251 & 0.0064 & -0.0048 \\
$\Lambda_{13} \times 10^{6}$ & 3.0 & 2.5 & -1.0 & 2.8 & 2.9 & -0.9 \\
$\beta_{1}(\mathrm{GeV})$ & 1.496 & 0.115 & -0.082 & 2.177 & 0.528 & -0.402 \\
$\beta_{2}(\mathrm{GeV})$ & 1.162 & 0.052 & -0.051 & 1.141 & 0.052 & -0.052 \\
$\beta_{3} \times \Lambda_{33}(\mathrm{MeV})$ & -431.162 & 0.070 & -0.040 & -431.145 & 0.065 & -0.035 \\
\hline$\beta_{3} \times 10^{-3}(\mathrm{GeV})$ & 5.8 & 2.6 & -1.9 & 4.8 & 2.6 & -1.8 \\
\hline
\end{tabular}

Table IV: $\chi^{2}$ values for the fits to the data sets 1 and 2 .

\begin{tabular}{|c|r|c|r|r|}
\hline $\begin{array}{c}\text { Set } \\
\text { No }\end{array}$ & $\begin{array}{c}\chi^{2} \\
\text { total }\end{array}$ & $\begin{array}{c}\chi^{2} \\
\delta \pi \pi \text { data }\end{array}$ & $\begin{array}{c}\chi^{2} \\
\varphi \text { data }\end{array}$ & $\begin{array}{c}\chi^{2} \\
\eta \text { data }\end{array}$ \\
\hline 1 & 75.2 & 52.2 & 6.7 & 16.4 \\
2 & 100.8 & 50.5 & 34.2 & 16.1 \\
\hline
\end{tabular}


Table V: Masses and widths of resonances obtained in fits to the data sets 1 and 2 compared with values of the Particle Data Group [34] and Ref. [39] for $f_{0}(500)$.

\begin{tabular}{|l|r|r|r|r|r|r|}
\hline \multirow{2}{*}{ pole } & \multicolumn{2}{|c|}{ set 1 } & \multicolumn{2}{c|}{ set 2 } & \multicolumn{2}{c|}{ Particle Data Group } \\
\cline { 2 - 7 } & $\mathrm{M}(\mathrm{MeV})$ & $\Gamma(\mathrm{MeV})$ & $\mathrm{M}(\mathrm{MeV})$ & $\Gamma(\mathrm{MeV})$ & $\mathrm{M}(\mathrm{MeV})$ & $\Gamma(\mathrm{MeV})$ \\
\hline$f_{0}(500)$ & $506 \pm 10$ & $494 \pm 5$ & $505 \pm 10$ & $497 \pm 5$ & $\leq 700$ & $\geq 600$ \\
$f_{0}(975)$ & $973 \pm 2$ & $29 \pm 2$ & $974 \pm 2$ & $30 \pm 1$ & $974.1 \pm 2.5$ & $47 \pm 9$ \\
$f_{0}(1400)$ & $1430 \pm 5$ & $145 \pm 25$ & $1428_{-7}^{+13}$ & $157_{-29}^{+43}$ & $\sim 1400$ & $150 \div 400$ \\
\hline
\end{tabular}

Table VI: Comparison of the $\pi \pi$ and $K \bar{K}$ scattering lengths obtained in the present work (sets 1,2 and nonrelativistic fit) with other determinations.

\begin{tabular}{|c|c|c|c|}
\hline Source & $a_{\pi \pi}\left(m_{\pi}^{-1}\right)$ & $\operatorname{Re}\left(a_{K \bar{K}}\right)(\mathrm{fm})$ & $\operatorname{Im}\left(a_{K \bar{K}}\right)(\mathrm{fm})$ \\
\hline set 1 & $0.172 \pm 0.008$ & $-1.73 \pm 0.07$ & $0.59 \pm 0.04$ \\
set 2 & $0.174 \pm 0.008$ & $-1.58 \pm 0.09$ & $0.61 \pm 0.04$ \\
nonrel. fit & 0.40 & -1.70 & 0.78 \\
48] & - & -1.15 & 1.80 \\
\hline [32] & $0.207 \pm 0.028$ & - & - \\
\hline
\end{tabular}




\section{FIGURE CAPTIONS}

- Fig. 1a. $K \bar{K}$ scattering phase shifts versus energy in the presence of the bound state at $975 \mathrm{MeV}$. Solid lines correspond to the relativistic $K \bar{K}$ propagator, while the dashed line to the nonrelativistic propagator. Two upper lines are calculated for $\beta_{1}=2000 \mathrm{MeV}$, two lower lines - for $\beta_{1}=150 \mathrm{MeV}$.

- Fig. 1b Same as in Fig. 1a but for the antibound state at $975 \mathrm{MeV}$.

- Fig. 1c $K \bar{K}$ scattering phase shifts versus energy in the presence of the resonance at $M_{s}=993 \mathrm{MeV}$ of the width $\Gamma=46 \mathrm{MeV}$. Both curves are calculated for the relativistic propagator: the upper one corresponds to $\beta_{1}=72 \mathrm{MeV}$ and $\Lambda_{11}=1.16$ and the lower one to $\beta_{1}=10.47 \mathrm{GeV}$ and $\Lambda_{11}=-0.14$.

- Fig. 2. Isospin $0 s$ - wave $\pi \pi$ phase shifts calculated for the data set 1 (solid line) and set 2 (dashed line). Arrows indicate the energy range used in the data fits. Data are from [28-31].

- Fig. 3. Sum of the $\pi \pi$ and $K \bar{K}$ phase shifts. Full circles denote data set 1 [43], open circles - data set 2 [44]]. Lines and arrows as in Fig. 2.

- Fig. 4. Inelasticity parameter $x=\left(1-\eta^{2}\right) / 4$. Data are taken from [43]. Lines and arrows as in Fig. 2.

- Fig. 5. Structure of the $S_{\pi \pi}$ matrix element in the complex $z$-plane. Positions of poles $\left(1-f_{0}(500), 2-f_{0}(975), 3-f_{0}(1400)\right)$ are indicated by crosses and zeros by circles. The roman numbers label the energy sheets. The bold line shows the physical region and the rectangles on the imaginary axis indicate the $S_{\pi \pi}$ cuts. 
This figure "fig1-1.png" is available in "png" format from: http://arxiv.org/ps/hep-ph/9403264v1 
This figure "fig2-1.png" is available in "png" format from: http://arxiv.org/ps/hep-ph/9403264v1 
This figure "fig1-2.png" is available in "png" format from: http://arxiv.org/ps/hep-ph/9403264v1 
This figure "fig2-2.png" is available in "png" format from: http://arxiv.org/ps/hep-ph/9403264v1 
This figure "fig1-3.png" is available in "png" format from: http://arxiv.org/ps/hep-ph/9403264v1 